\title{
Economía circular y su sostenibilidad ambiental, económica y social en comunidades de Barranquilla, Atlántico, Colombia.
}

\author{
${ }^{\text {a }}$ Rafael Oyaga Martínez, ${ }^{\mathrm{b}}$ Daniela Martínez Quinto, ${ }^{\mathrm{c}}$ Pedro Pacheco Torres, ${ }^{\mathrm{c}}$ Sofía Brisseth Sulbaran Siado \\ \& ${ }^{\mathrm{e}}$ Janneris Rodríguez Gómez \\ ${ }^{a}$ Grupo CRIDI, Corporación Universitaria Reformada. \\ r.oyaga@unireformada.edu.co \\ ${ }^{b}$ Programa de Administración marítima y portuaria, Corporación Universitaria Reformada. \\ daniela.martinez@unireformada.edu.co \\ ${ }^{c}$ Programa de Ingeniería Ambiental, Corporación Universitaria Reformada. \\ ing.ambiental@unireformada.edu.co,s.sulbaran@unireformada.edu.co \\ ${ }^{d}$ Decanatura de Facultad de Ingeniería, Corporación Universitaria Reformada. \\ decanaturas.ingenierias@unireformada.edu.co
}

Recibido: Marzo 04, 2021.

Recibido en su versión corregida: Abril 23, 2021.

https://doi.org/10.54606/Sextante2021.v24.04

Aceptación: Mayo 07, 2021.

Cómo citar: Oyaga Martínez, R., Martínez Quintero, D., Pacheco Torres, P., Sulbaran Siado, S. \& Rodriguez Gómez, J. (2021). Economía circular y su sostenibilidad ambiental, económica y social en comunidades de Barranquilla, Atlántico, Colombia. Revista Sextante, 24, pp. 29 - 33, 2021.

\section{Resumen}

Se presenta el trabajo de investigación desarrollado en el año 2019 y con proyección al 2020, que busca evaluar la viabilidad técnico-económica y social de la implementación de empresas de clasificación y aprovechamiento de residuos, partiendo de la regulación vigente. Desde este enfoque se desea desarrollar una serie de procesos con las comunidades de recicladores de Barranquilla, con el fin de implementar estrategias que permitan establecer la sostenibilidad de las empresas constituidas para el desarrollo de la economía circular en el marco del decreto 0596 de 2016 y su transición al decreto 2412 de 2018. Se reporta ausencia de cultura organizacional, riesgos asociados a la actividad de reciclaje, incumplimiento de las etapas de formalización.

Palabras clave: Aprovechamiento, Formalización, Negocio, Recicladores, Reciclaje.

Circular economy and its environmental sustainability, economic and social sustainability in communities of Barranquilla, Atlántico, Colombia.

\begin{abstract}
The research work developed in 2019 and with a projection to 2020 is presented, which seeks to evaluate the economic and social-technical feasibility of the implementation of waste classification and use companies based on regulation; from this approach, we want to develop a series of processes with the communities of recyclers in Barranquilla in order to implement strategies that allow establishing the sustainability of the companies incorporated for the development of the economy circular under decree 0596 of 2016 and its transition to decree 2412 of 2018. Reports absence of organizational culture, the risk associated with recycling activity, non-compliance with the stages of formalization.
\end{abstract}

Keywords: Business, Formalization, Recyclers, Recycling, Utilization. 


\section{Introducción}

Entre los objetivos del desarrollo sostenible se destaca el número 11, que plantea: las ciudades son hervideros de ideas, comercio, cultura, ciencia, productividad, desarrollo social y mucho más. En el mejor de los casos, las ciudades han permitido a las personas progresar social y económicamente. En los últimos decenios, el mundo ha experimentado un crecimiento urbano sin precedentes.

En 2015, cerca de 4.000 millones de personas vivían en ciudades y se prevé que ese número aumente hasta unos 5.000 millones para el 2030. Se necesita mejorar, por tanto, la planificación y la gestión urbana para que los espacios urbanos del mundo sean más inclusivos, seguros, resiliente y sostenibles.

Ahora bien, son muchos los problemas que existen para mantener ciudades, de manera que se sigan generando empleos y siendo prósperas, sin ejercer presión sobre la tierra y los recursos. Los problemas comunes de las ciudades son la congestión, la falta de fondos para prestar servicios básicos, la falta de políticas apropiadas en materia de tierras y vivienda y el deterioro de la infraestructura. La economía circular proporciona múltiples mecanismos de creación de valor no vinculados al consumo de recursos finitos.

En una verdadera economía circular el consumo solo se produce en ciclos biológicos eficaces; por lo demás, el uso sustituye al consumo. Los recursos se regeneran dentro del ciclo biológico o se recuperan y restauran gracias al ciclo técnico. Dentro del ciclo biológico, distintos procesos permiten regenerar los materiales descartados, pese a la intervención humana o sin que esta sea necesaria. En el ciclo técnico, con la suficiente energía disponible, la intervención humana recupera los distintos recursos y recrea el orden, dentro de la escala temporal que se plantee. Mantener o aumentar el capital supone características diferentes en ambos ciclos.

La economía circular se basa en tres principios clave, cada uno de los cuales aborda varios de los retos en términos de recursos y del sistema a los que han de hacer frente las economías industriales. Vivir de forma sostenible no es simplemente un estilo de vida, o un problema ecológico.
Al abordar nuestros desafíos sociales y ecológicos con soluciones prácticas, también tenemos que explorar las raíces psicológicas y espirituales de estos desafíos para poder crear de manera colectiva una cultura humano-sostenible para todos.

En una Estación de Clasificación y Aprovechamiento (ECA) se almacenan los materiales aprovechables después de uso y consumo, pertenecientes a los grupos de papel y cartón, tales como: archivo, periódico, cartón, plegadiza; también los ubicados en plásticos, como: los envases PET, pasta, bolsas plásticas de baja y alta densidad completamente "sostenible", pero la búsqueda de proyectos y comunidades que estén trabajando hacia la sostenibilidad desde un enfoque holístico, sabiendo que estos modelos y ejemplos pueden ayudar a catalizar un movimiento mayor hacia una vida sostenible en una escala masiva.

Entonces, bajo el contexto anteriormente planteado es necesario realizar el siguiente interrogante:

¿Cómo se puede desarrollar un proyecto sostenible desde lo ambiental, social y económico en las comunidades de recicladores involucradas, para el desarrollo de una empresa de clasificación y aprovechamiento de residuos, de acuerdo con la regulación vigente?

\section{Referente teórico}

La economía circular se encuentra implícita desde la cumbre de Rio en donde se planteó el componente medio ambiental como elemento constitutivo del ámbito económico-empresarial (Balboa y Domínguez, 2013), en el artículo los autores plantean igualmente que en general se le puede considerar como una filosofía de diseño de negocio.

El modelo de economía circular es, en su contexto, un modelo de negocio direccionado hacia la construcción de un nuevo paradigma que tiene implicaciones de construcción de formas de negocios, atendiendo el nivel de crecimiento económico de la comunidad en la cual el proyecto este inserto, ello implica una nueva modalidad de hacer productos desde su mismo origen, diseño y 
nivel de aprovechamiento de las materias primas y recursos energéticos (Lett, 2014).

\section{Metodología}

El desarrollo de esta investigación se realizará en seis etapas, como se muestra en la Figura 1.

Se inició con una revisión bibliográfica y legal para la etapa 1, además se realizó un reconocimiento del territorio mediante la visita a las zonas de influencia de las ECAS intervenidas; y se recolectará información a través de diferentes fuentes para el diagnóstico de la etapa 2. Para la recolección de la información se diseñaron tablas (relación de documentos y análisis de estos).

En estas herramientas se relacionaron documentos como: el plan de Ordenamiento Territorial en relación con el manejo integral de residuos, el Plan de Desarrollo distrital, la Política Nacional de Economía Circular, el Plan de Manejo Integral de Residuos Sólidos, encuestas y entrevistas a participantes y validación de la información.
En la etapa 3 los equipos de trabajo proyectaron planes de acción para la migración de las ECAS a empresas que puedan ejecutar el esquema de reciclaje presente en el decreto 0596 de 2016. La etapa 4 evaluará el modelo de negocio con todos los actores del proceso, para la viabilidad económica del montaje de esta empresa.

El análisis de tipo social se ejecutará en la etapa 5, durante este proceso identificaremos las limitaciones de las comunidades que actuarán en este proceso. Finalmente ejecutaremos proyecciones ambientales con el objetivo de diagnosticar la disminución de emisiones y los cambios en las estructuras de reciclaje.

\section{Resultados}

Durante el desarrollo de este proyecto iniciamos con procesos de intervención dentro 3 diferentes asociaciones de las 17 ECAS existentes en la ciudad de Barranquilla, con las que se trabajarán.

La Figura 2 muestra las instalaciones de la ECA Metroreciclar.

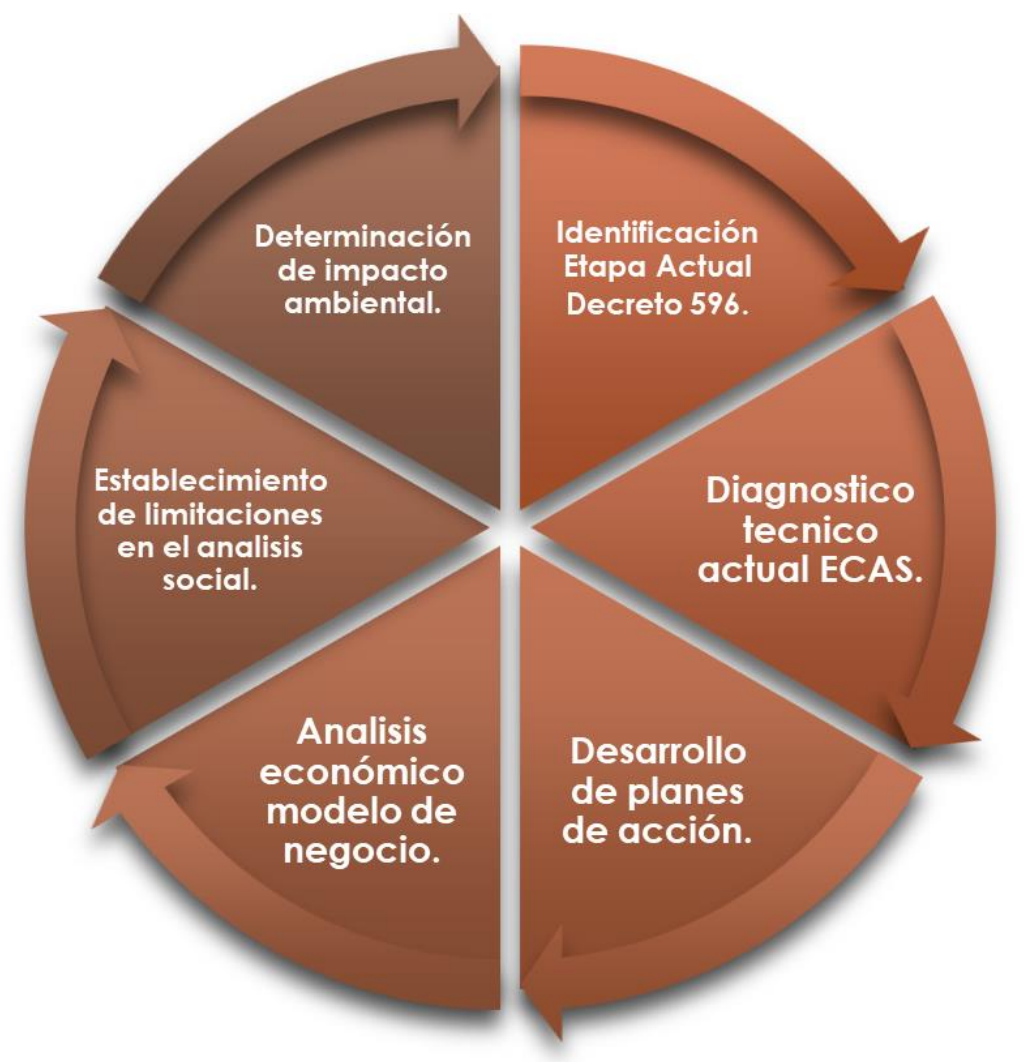

Figura 1. Etapas de la investigación.

Fuente: Los autores. 

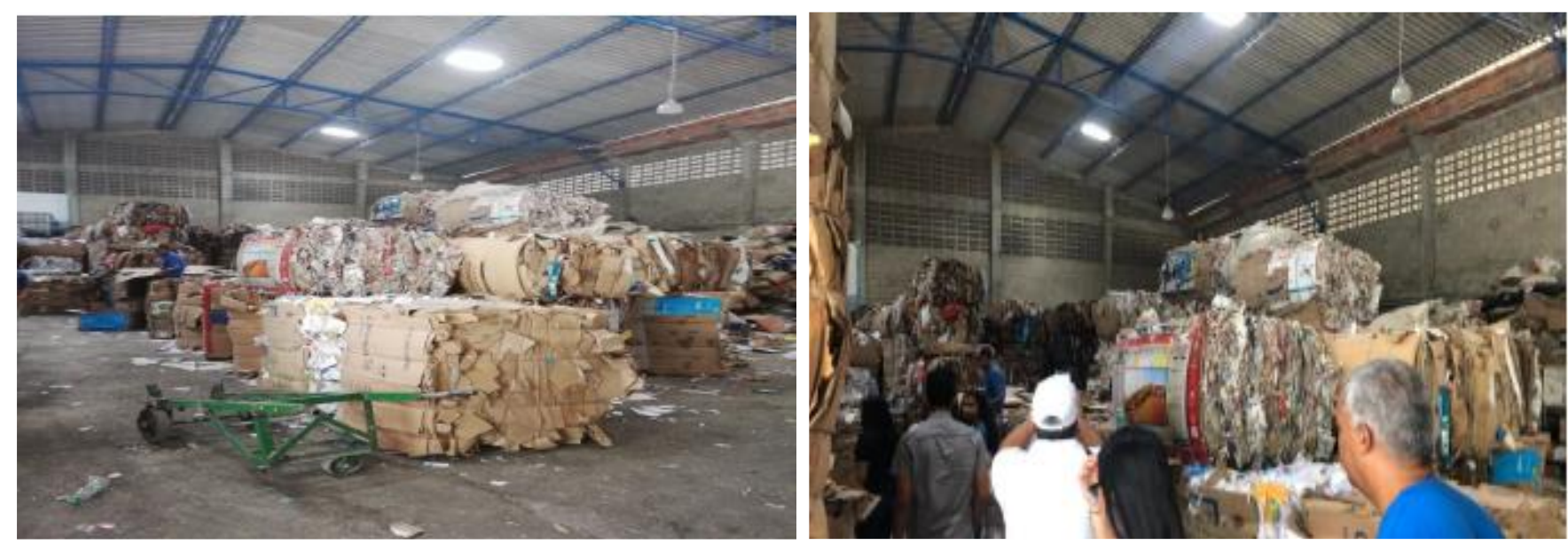

Figura 2. ECA Metroreciclar.

Fuente: Tomado de Metroreciclar, (2019).
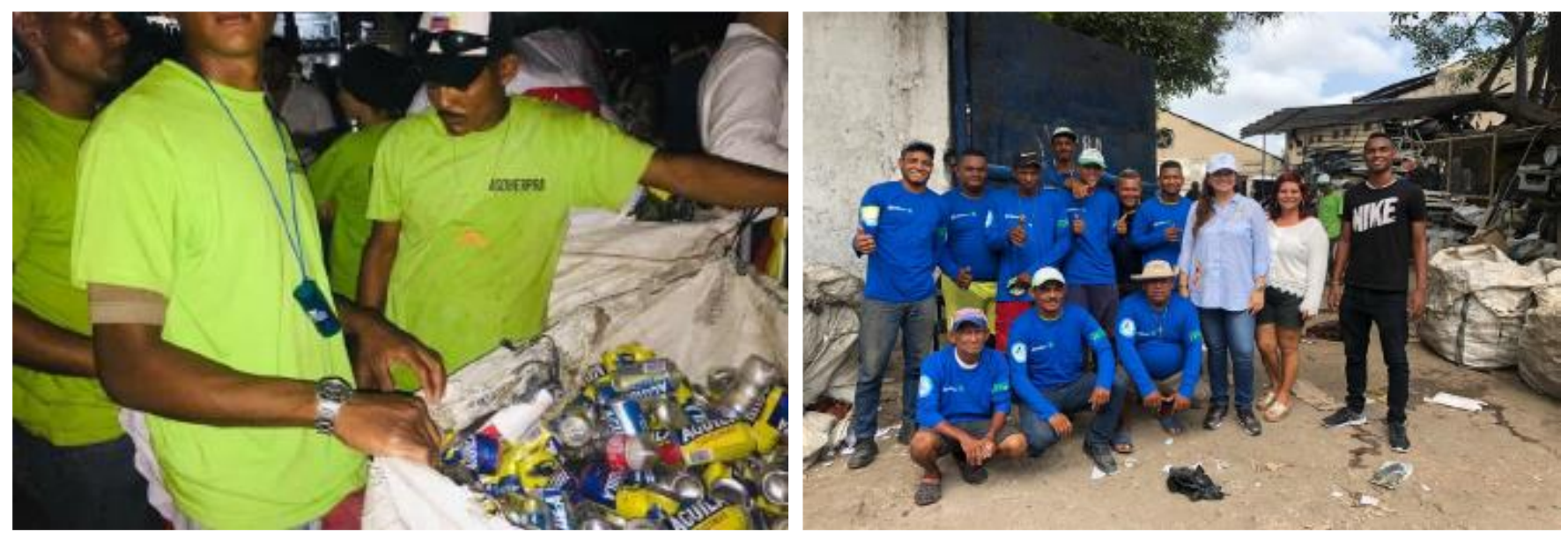

Figura 3. ECA Asoherpra.

Fuente: Los autores.

La ECA Metroreciclar cuenta con 150 recicladores registrados, dentro de su misión se identifican como "una institución prestadora del servicio de recolección, transporte, clasificación y comercialización de material aprovechable, que trabaja en la formación integral, gestión y reconocimiento del trabajo realizado por los recicladores de oficio, buscando su bienestar y óptimas condiciones de trabajo" (Metroreciclar, 2019). La Figura 3 muestra una parte de los miembros de la ECA Asoherpra.

La ECA Asoherpra cuenta con 72 recicladores registrados, esta es dirigida por la señora Hersilia Prada, que cuenta con más de 15 años de experiencia en este sector. En la Figura 4 encontraremos los equipos con los que cuenta la ECA Asobapre.

La ECA Asobapre, que cuenta con 140 recicladores registrados, es dirigida por el señor Augusto Picón. Esta asociación, que está ubicada sobre la calle 17, es un referente para el desarrollo de los recicladores.

\section{Conclusiones}

Como conclusiones del trabajo de investigación se involucran los siguientes aspectos:

Frente a los costos de infraestructura y logísticos: Se encontró que hay limitados recursos económicos $\mathrm{y}$ hacen falta mayores incentivos e inversiones estratégicas. Se presenta una situación asociada a altos costos logísticos.

En cuanto a las alianzas público-privadas se denota una marcada ausencia de colaboración público-privada, hay que reforzar en todos los puntos una seguridad jurídica al inversor. Igualmente existen dificultades con los recicladores en la creación de las asociaciones. 

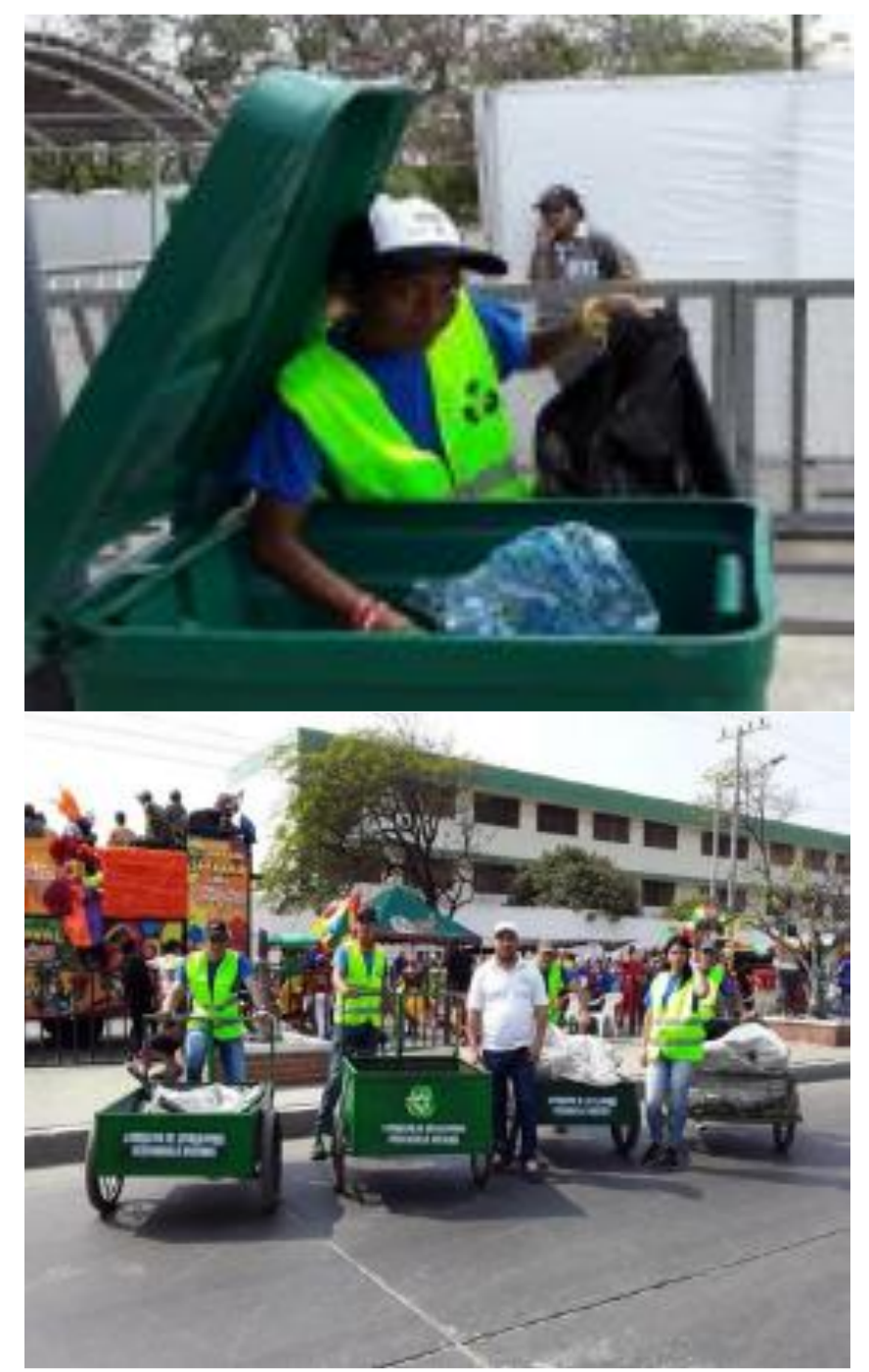

Figura 4. ECA Asobapre.

Fuente: Los autores.

En cuanto a los instrumentos económicos, se encontró una marcada deficiencia en los esquemas de financiación dirigidos a las regiones y municipios, se requiere fortalecer los incentivos a la inversión privada en infraestructura y logística.

Además, hay una marcada ausencia de programas de subvenciones. También existe atraso en los procesos de los pagos tarifarios.

En conclusión, existe en los mercados fallas de logística inversa y suministro, además de una elevada informalidad en la recogida de recursos.

\section{Referencias}

Balboa, C. H., \& Somonte, M. D. (2014). Economía circular como marco para el ecodiseño: el modelo ECO-3. Informador técnico, 78(1), 8290.

Benyus M., J. (2003). Biomimicry: Innovation Inspired by Nature,Editor William Morrow. ISBN: 978-00-6053-322-9

Ellen Macarthur Fundation: Report. (2013). Towards the Circular Economy. Recuperado de http://www.ellenmacarthurfoundation.org/circul ar-economy/circulareconomy/the-circularmodel-an-overview

Ellen Macarthur Fundation: Report. (2013). Work towards using energy from renewable sources. Recuperado de http://www.ellenmacarthurfoundation.org/circul ar-economy/circular-economy/the-circularmodel-an-overview

Ehrenfeld, J., Gertler, N. (1997). Industrial ecology in practice:the evolution of interdependence at alundborg. Journal

Hawken P., Lovins A., Lovins L. (2000). Natural Capitalism:Creating the Next Industrial Revolution. Nueva York, Back Bay Books. ISBN: 978-03-1635-300-7

Lyle T., J. (1994). Regenerative Design for Sustainable Development. Editorial Wiley and Sons, Nueva York. ISBN:978-04-7155-582-7

Metroreciclar, (2019). Pagina de presentación Metroreciclar - Misión, recuperado de: http://www.metroreciclar.com/, acesso: 15 de septiembre de 2019

Scientific American, 144-152. Goleman, D. (1999). La práctica de la inteligencia emocional. Barcelona, Editorial Kairós. ISBN: 978-847245-407-1 